II Congreso Internacional Estéticas Híbridas de la Imagen en Movimiento: Identidad y Patrimonio

\title{
Cuerpo, límites y autobiografía: Una aproximación a lo sonoro en la obra de Marina Abramović
}

\author{
Úrsula San Cristóbal ${ }^{1}$ \\ ${ }^{1}$ Videoartista e investigadora, docente en Taller de Músics Escuela Superior de Estudios \\ Musicales, usancristobal@gmail.com
}

\begin{abstract}
This text proposes a first approach to the role of sound in Marina Abramović's solo work, through a comparison between her pieces from the 1970s and those from the 1990s. First, some of the sound installations by the artist are addressed, which show an incipient relationship between sound and performance. Second, the sonic elements in the Rhythm series (1973-74) are discussed, showing how they collaborate with the exploration of the limits of the body. Finally, the performances Delusional (1994) and Balkan Baroque (1997) are addressed, highlighting how Abramović goes from sound to music when she begins to focus on her autobiography.
\end{abstract}

Keywords: Marina Abramović; sound; performance art; video art.

\section{Resumen}

Este texto propone una primera aproximación al rol de lo sonoro en la obra en solitario de Marina Abramović, a través de una comparación entre sus piezas de la década de 1970 y las de 1990. En primer lugar se abordan algunas de las instalaciones sonoras de la artista que muestran una relación incipiente entre el sonido y la performance. En segundo lugar se comentan los elementos sonoros en la serie Rhythm (1973-74), mostrando cómo colaboran con la exploración de los límites del cuerpo. Por último, se abordan las performances Delusional (1994) y Balkan Baroque (1997), poniendo en evidencia cómo Abramović pasa del sonido a la música cuando comienza a centrarse en su autobiografía.

Palabras clave: Marina Abramović, sonido, performance art, videoarte 


\section{Introducción}

Los elementos sonoros son una constante en el trabajo de Marina Abramović, aunque su presencia ha sido objeto de escuetos comentarios en la bibliografía especializada. Los pocos textos que se refieren al tema abordan principalmente su obra temprana de la década de 1970. Autoras como (Iles, 1995)? y (Biesenbach, 2010)?, solo se refieren al sonido como temporalidad en las instalaciones y performances realizadas en Belgrado. (LaBelle, 2006, 2014) y (Hegarty, 2015) por su parte, se refieren de manera muy breve al uso de la voz en piezas de video performance, pocos escritos abordan obras posteriores. (Richards, 2010)? menciona la presencia de canciones populares en las obras de la década de 1990, pero las referencias suelen ser tan generales que en ocasiones resulta imposible determinar qué músicas fueron empleadas. Cómo se relacionan el sonido, la escucha y el cuerpo, y qué rol cumple la música en el desarrollo conceptual de las performances de Abramović, constituyen interrogantes aún por explorar.

En este texto abordo una aproximación inicial al rol de lo sonoro en la obra en solitario de Marina Abramović. En primer lugar, me refiero brevemente a algunas de las primeras instalaciones sonoras de la artista que muestran cómo a través del sonido comienza a abordar conceptos que serán centrales en sus performances. En segundo lugar, aporto un comentario sobre lo sonoro en la serie Rhythm (1973-74), mostrando los vínculos entre el sonido, la escucha y la exploración de los límites del cuerpo. Finalmente me refiero a las performances Delusional (1994) y Balkan Baroque (1997), poniendo en evidencia cómo Abramović pasa del sonido a la música cuando comienza a centrarse en su autobiografía.

\section{Antecedentes: Sonido, violencia, calma y vacío}

La relación de Abramović con el sonido se remonta a sus primeros trabajos realizados en Belgrado. A través del formato de la instalación sonora comenzó a abordar conceptos como el vacío (Sound Environment - White, 1972), la repetición y la temporalidad (Spaces, 1974). De este período es interesante destacar las piezas Sound Corridor/War y Forest, ambas realizadas para la muestra Young Artists and Young Critics en el Museo de arte contemporáneo de Belgrado en febrero de 1972. En ellas se explora el contraste entre la calma y la violencia, rasgos que reaparecen en algunas de sus performances en solitario. En Sound Corridor/War (Zvučni ambijent rat) el público debía atravesar un corredor vacío antes de acceder al espacio expositivo. Al entrar en el corredor, la audiencia era sorprendida por los sonidos de una ametralladora que colmaban el espacio (Biesenbach et al, 2010; p. 54; Richards 2010) ]. Al salir del corredor el público experimentaba el silencio. Esta experiencia parecía preparar a la 
audiencia para la instalación Forest, donde resonaban sonidos del bosque (viento, animales, etc.). En una pared cubierta de papel blanco se incluían las siguientes instrucciones: "Walk, run, breathe. Feel like you are in the forest. Write your impresions" (Biesenbach et al, 2010, p. 59; Westcott, 2010, p. 57)?]? Iles interpreta el contraste entre ambas piezas a la luz de una tradición budista frecuentemente mencionada por Abramović: sentarse bajo una cascada durante un tiempo prolongado, para luego abandonar el lugar y experimentar el silencio de una manera completamente diferente (Biesenbach, 2016; Iles, 1995, p. 22)]. Saturar la escucha para luego liberar la mente, un principio que se manifiesta más tarde en algunas de sus primeras performances.

Sound Corridor/War muestra además otra característica: el sonido ocupa al espacio sustituyendo a los objetos materiales y se comporta como una fuerza capaz de impactar a la audiencia, incluso al extremo de ejercer una violencia invisible sobre sus cuerpos. Esta violencia sonora puede haber tenido como antecedente otro proyecto de Abramović, en el cual reproducía el sonido de un derrumbe en un espacio público. La descripción de esta pieza se confunde entre los datos disponibles y el recuerdo de la propia artista. Según la información aportada por el Centro cultural de estudiante de Belgrado (Studentski Kulturni Centar, en adelante SKC), esta pieza se habría titulado Ozvučeni prostor-rušenje fasade (espacio sonoro - demolición de fachada) y habría sido realizada para la muestra Oktobar 72 (SKC, 2009; Westcott, 2010, p.57) 国. El sonido habría sido reproducido en la fachada del SKC, dando la impresión de que el edificio se derrumbaba sobre los transeúntes. Sin embargo, Abramović en una entrevista para The Guardian expone una versión diferente. Indica que pretendía realizar la pieza sobre un puente, pero al no obtener los permisos necesarios, habría decidido reproducir el sonido del derrumbe en el edificio en que vivía, lo que habría generado conmoción entre habitantes y peatones:

Suddenly the people, they are rushing out into the streets, freaking out everywhere, thinking they are being bombed. For me... this was an incredible thing. I realise the power of art that does not hang on the walls of galleries. (Abramovic entrevistada en O'Hagan, 2010)?

Esta versión resulta más impactante que la reportada en las fuentes, y sugiere la necesidad de Abramović de mostrarse como una artista que ya desde su juventud buscaba desconcertar. Tanto en esta pieza como en Sound Corridor/War se aprecia un interés por perturbar al público, algo que es posibilitado por el empleo de un medio artístico como el sonido, considerado no convencional en la época. Esto puede entenderse como una manifestación temprana de la exploración de los límites del cuerpo que Abramović desarrollará en su primera serie de performances, donde agredir el propio cuerpo es también una manera de confrontar a la audiencia. 
Cuando Abramović pasó de las instalaciones a la performance, el sonido fue un elemento de continuidad. Al sonido pregrabado se sumaron los sonidos producidos in situ por el cuerpo del artista y por los asistentes, tal como se aprecia en la serie titulada Rhythm, un conjunto de cinco performances (10, 5, 2, 4 y 0 ) realizadas en solitario entre 1973 y 1974, en las que se manifiestan dos de los temas más característicos de la artista: el riesgo y la exploración de los límites del propio cuerpo.

\section{Del sonido al cuerpo: La serie Rhythm}

"After the sound work, there was a natural development toward work with the body [...] The first performance with the body was Rhythm 10 - the work with the knives" (Brayshaw \& Witts, 2013, p. 10)]. Rhythm 10 puede considerarse la pieza que inaugura la exploración del riesgo en la obra de Abramović, y lo hace profundizando en la necesidad de perturbar a la audiencia que ya se venía prefigurando en instalaciones como Sound Corridor/War. Refiriéndose a este tipo de acciones Abramović ha declarado: "I'm interested in art that disturbs and that pushes that moment of danger" (Abramović et al, 1998, p. 44)? Pero Rhythm 10 no es solo una exploración del peligro. También presenta un interesante uso del sonido y de la grabación in situ, lo que sugiere una relación entre la investigación sonora y el cuerpo.

La acción se realizó en dos ocasiones en 1973, primero en el Festival de Edimburgo y luego en el Museo de Arte Contemporáneo de la Villa Borghese en Roma. De acuerdo con la descripción del catálogo, la artista trabajó con los siguientes materiales: una hoja de papel blanco de grandes dimensiones sobre el suelo, 20 cuchillos (10 en la presentación en Edinburgo), y dos grabadoras (Biesenbach et al, 2010, p. 60)? Abramović apoyó la palma de su mano izquierda contra el suelo y con la derecha comenzó a clavar uno de los cuchillos con rapidez en el espacio en medio de los dedos. Mientras tanto, registraba el sonido de la acción con una de las grabadoras. Cada vez que se hacía un corte, cambiaba de cuchillo. Una vez usados todos los cuchillos, la artista hizo una pausa para escuchar la grabación. Luego repitió la acción sobre la primera grabación, intentando replicar todas las veces en que se cortó ("mistakes" según la artista), registrando el sonido con la segunda grabadora. Finalmente reprodujo el sonido de ambas grabadoras y abandonó el recinto. Según Abramović: "In this performance, the mistakes of the past and those of the present are synchronous." (Biesenbach et al., 2010, p. 60)?]. Esta ha sido la interpretación más difundida de la pieza. Sin embargo, en una monografía sobre las obras de juventud de Abramović publicada en 2013, el autor Olivera Jankovic aporta más información sobre la versión realizada en Edimburgo:

There are indications that in the course of the performance, she had by her a photograph of herself as a toddler, accompanied by her father in military uniform, walking in the old fort of Kalemegdan in Belgrade. This was a biographical element 
that would have given the work the dimension of an intimate experience. To music randomly selected from the radio, she first painted the nails of her left hand blue, then turned off the radio and turned on the cassette recorder to capture the sound of the next part of the performance. (Jankovic, citado en Richards, 2018)

Una acción que pone al cuerpo en situación de riesgo a través de elementos que remiten a la infancia, a la intimidad y a la cultura de origen de la artista. De acuerdo con su relato, la acción de los cuchillos se inspiraría en un juego de campesinos rusos y Yugoslavos: "Like Russian roulette, it is a game of bravery and foolishness and despair and darkness-the perfect Slavic game" (Abramović, 2016)国. Sin profundizar en lo problemática que resulta esta referencia a lo Eslavo, creo que refleja de manera incipiente el tipo de vínculo que Abramović establece entre sus acciones artísticas y lo autobiográfico, donde la región de origen acaba siendo objeto de consideraciones arquetípicas, un aspecto que vuelve a presentarse en piezas como Delusional y Balkan Baroque.

En cuanto a lo sonoro, Rhythm 10 podría considerarse la primera pieza de la artista en que aparecen juntos el cuerpo, el sonido y el tiempo, algo que ha sido reconocido por la artista en algunas entrevistas (Biesenbach, 2016) ${ }^{1}$. El tiempo es un elemento fundamental en Abramović y comenzó a ser explorado gracias al sonido. De hecho, se puede llegar a suponer que experimentar con la relación entre tiempo y sonido contribuyó a la exploración de los límites del cuerpo. Al recurrir al sonido, Abramović no solo estaba explorando más allá de lo visual. También estaba indagando en la capacidad de su cuerpo para llevar a cabo una acción riesgosa durante un tiempo prolongado. El sonido rítmico del cuchillo focaliza su concentración mental y física en el momento presente, comportándose de manera similar al sonido del metrónomo que se aprecia en instalaciones sonoras como Spaces (1974), pero con la diferencia de que en Rhythm 10 es la propia artista la que produce el sonido. Mary Richards ha destacado la concentración y el control como elementos característicos de esta acción:

While suspense mounted as neither spectator nor Abramović knew where or when the knife would fall and cut, halfway through the performance Abramović takes control over these apparently random events and re-inscribes the cutting by a close reproduction of her original actions during the first half of the performance. This was a performance game carefully set up and her ability to carry out these actions and re-do them with such a high degree of precision was evidence of her capacity to

1 "[KB]: Can you remember when you started visualizing or expressing time as sound? I think this is quite an important characteristic of your work.

[MA]: I think I did this most consciously in Rhythm 10 (1973)." (Biesenbach, 2016)]. 
Cuerpo, límites y autobiografía: Una aproximación a lo sonoro en la obra de Marina Abramović

endure and control events in a way that was personally empowering. (Richards, 2018)

En esta pieza la concentración va ligada a la escucha. Para repetir la acción la artista necesitó escuchar la grabación. Tuvo que poner atención al ritmo y al cambio que ocurría en el sonido cada vez que se cortaba, y es probable que la escucha ayudara a la artista a alcanzar un cierto estado de concentración necesario para mantener el control sobre la acción. Abramović suele definir la performance como un estado mental, articulado como una estructura física y mental de intercambio de energías con el público, resumida en la idea de "performance field" (véase lles, 1995, p. 32; Westcott, 2010 , p. 210). Resulta relevante considerar cómo su trabajo con el sonido pudo haber contribuido a forjar esta concepción particular de la performance.

Como bien observa Richards, en el resto de la serie Rhythm el control comenzó a desaparecer: "The later Rhythm performances [...] were deliberately experimenting with limiting or removing Abramović's control over the outcome of events" (Richards, 2010, p. 85)?]. En el resto de la serie (Rhythm 5, 2, 4 y 0) el sonido ya no fue producido por la artista sino por el público y los objetos que intervenían en la acción. Esto sugiere una relación entre el sonido, la escucha y el control en esta primera etapa de Abramović. Cuando la artista deja de producir sonidos repetitivos, la concentración se sitúa en la escucha del entorno sonoro que se genera durante la performance. Esto se aprecia en Rhythm 5, pieza realizada en el SKC en el marco de los III Encuentros de abril de 1974. Abramović trazó una estrella de cinco puntas en el suelo y le prendió fuego. Luego cortó su cabello y sus uñas y las lanzó a las llamas, y finalmente se recostó de espaldas en el centro de la estrella. Al cabo de un tiempo, la artista perdió el conocimiento por la falta de oxígeno y fue rescatada por un par de colegas. La pieza tiene un carácter de sacrificio ritual y según la artista, la estrella de cinco puntas representaría el poder opresivo del comunismo, al mismo tiempo que haría referencia a su simbolismo esotérico asociado al pentagrama del ocultismo ${ }^{2}$.

2 "Why a star? it was the symbol of Communism, the repressive force under which I had to grow up, the thing I was trying to escape-but it was so many other things, too: a pentagram, an icon worshiped and mystified by ancient religions and cults, a shape possessing enormous symbolic power." (Abramović 2016) Hay que considerar que Abramović no era precisamente una víctima del régimen. Como observa Richards, el hecho de que Abramović pudiera realizar este tipo de acciones en un espacio público sin ser arrestada muestra su grado de libertad (Richards, 2010, p. 2). La supuesta crítica política de Rhythm 5 también ha sido discutida por el crítico serbio Nikola Pešić, quien menciona la favorable acogida que tuvo la obra dentro de las instituciones comunistas del período. También indica cómo las publicaciones en inglés habrían contribuido a difundir la idea de esta obra como una crítica al comunismo (Pešić, 2018, p. 245). 
Lo que me interesa remarcar de esta pieza es la ausencia de control. La artista no pudo controlar la reacción de su cuerpo ni la de los miembros de la audiencia que fueron en su ayuda. Tampoco pudo controlar los sonidos producidos por el fuego ni los producidos por la audiencia. Su atención parecía estar en la escucha, tal como lo describe en su recuerdo de Rhythm 5: "There was a dead silence-all you could hear in the courtyard was the crackling of the flames. That was the last thing I remembered" (Abramović 2016). La falta de control fue más allá de la voluntad de la artista y en varias entrevistas ha señalado cuán molesta estaba de que el público hubiese interrumpido la acción (Lund, 2017), algo que volvió a ocurrir un año más tarde en la bien conocida pieza Lips of Thomas (1975), donde el público decidió poner fin a la auto flagelación de la artista. Cabe destacar que a pesar de la opinión negativa de Abramović, la intervención de la audiencia fue uno de los momentos más importantes de esta Rhythm 5 y que ha sido más valorado por la crítica y la teoría, ya que el público dejó de observar para tomar un rol activo en la performance. La pérdida de control por parte de la artista permitió que la audiencia tomara sus propias decisiones y se integrara a la obra (véase Fischer-Lichte, 2011, pp. 25-26).

El objetivo de Abramović era llevar el cuerpo al límite sin que nadie pudiese evitarlo, e intentó ponerlo en práctica en las siguientes piezas de la serie. En Rhythm 2 (1974) realizada en la Galeria Suvremene Umjetnosti de Zagreb, la artista tomó dos medicaciones, una para la catatonia y otra para la esquizofrenia. La primera provocó espasmos involuntarios en su cuerpo, mientras que la segunda inhibió por completo sus reacciones. A modo de intermedio entre ambas medicaciones, Abramović encendió una radio y la sintonizó en una estación al azar (Véase Biesenbach et al, 2010, pp. 68-69; Richards, 2010, p. 87). En esta pieza el control no funcionó como se había previsto. La artista no tuvo control sobre su cuerpo por causa de la medicación, del mismo modo que no tuvo control sobre los sonidos emitidos por la radio durante el intermedio. ${ }^{3}$ Como ya he mencionado, a menos control, más protagonismo adquieren los sonidos del entorno. En este sentido, la radio sintonizada en tiempo real puede interpretase como un medio que reforzaba el "aquí y ahora" de la performance, y enfatizaba el paso del tiempo para la audiencia.

En la siguiente pieza la artista intentó recuperar el control recurriendo al uso del espacio y al vídeo. Rhythm 4 (1974) realizada en la Galleria Diagramma en Milán, es la única pieza de la serie que emplea el circuito cerrado de televisión. La artista empleó

3 En algunas entrevistas Abramović indica que en esta acción experimentó descontrol con la primera pastilla y control con la segunda, algo que es cuestionada por Richards: “... once the pills has been taken ... and the drug had reached her bloodstream then the way her body reacted was in certain fundamental ways, beyond her control; that is, the substance of the pill itself effectively overrode Abramović's ability to control it" (Richards, 2010, p. 87). 
dos salas, en una realizaba la performance y en la otra estaba el público viéndola a través de un monitor de video. Abramović empleó un ventilador industrial, se puso de rodillas frente a él e intentó respirar todo el aire que fuera posible. La cámara enfocaba su cabeza por lo que el público sólo pudo presenciar la deformación facial que la presión del aire provocaba en su rostro. A un cierto punto la artista perdió el conocimiento por causa de la hiper ventilación, pero la presión del aire la mantuvo suspendida. Según Abramović, en este momento se cumplió el objetivo de la pieza: "The moment I lose consciousness the performance lasts 3 more minutes, during which the public are unaware of my state. In the performance I succeed in using my body in and out of consciousness without any interruption" (Biesenbach et al, 2010, p. 72). No he podido acceder a la documentación audiovisual de esta pieza, por lo que solo puedo suponer cómo era la sonoridad. El sonido constante y homogéneo del ventilador probablemente saturó la escucha de la artista, comportándose de manera similar al sonido de la cascada en el relato budista mencionado antes. Es posible pensar el sonido en Rhythm 4 como un proceso de saturar la escucha para así renovar la percepción. Este sería un proceso que afectaría principalmente a la artista ya que la audiencia no podía escuchar el sonido en directo.

En síntesis, en el desarrollo de la serie Rhythm se aprecia el paso de la producción controlada de sonidos a la escucha del ambiente: el sonido deja se ser un elemento que da ritmo y dirección a las acciones y se vuelve un objeto de escucha, que permite percibir el propio cuerpo y el entorno. Se trata de escuchar aquello que no se puede controlar, de percibirlo y experimentar el paso del tiempo. Esta escucha fomenta el desarrollo del estado mental que Abramović considera necesario para la performance, y probablemente contribuyó a llevar a cabo la pieza final de la serie. En Rhythm 0 (1974) realizada en el Studio Morra en Nápoles, la artista presentó su cuerpo junto a una serie de 72 objetos que podían ser usados para proporcionar placer o dolor, permitiendo al público hacer lo quisiera durante seis horas ${ }^{4}$.

Por otra parte, la presencia del sonido grabado en piezas como Rhythm 10 invita a replantearse la idea de la performance entendida exclusivamente como arte efímero. Si el sonido de la acción se reproduce en el trascurso de la misma, ésta deja de concebirse como presente perpetuo para entrar en diálogo con su propia documentación. La tensión entre inmediatez, documentación y re-presentación continuará presente en el trabajo de Abramović y planteará debates incluso más complejos, como se aprecia en la muestra Seven Easy Pieces, donde la artista re-presentó piezas celebres de la historia de la performance.

4 Lamentablemente no he podido consultar documentación audiovisual sobre esta acción que me permita analizar el entorno sonoro. 
Las obras mencionadas hasta aquí muestran como las exploraciones sonoras de Abramović se enmarcaban en una búsqueda similar a la de otros artistas del período. Por ejemplo, la relación entre sonido, espacio y temporalidad, y el uso de la grabación y su reproducción en acciones como Sound Environment White (1972) y Rhythm 10 (1973), las encontramos en obras como / am Sitting in a Room de Alvin Lucier. La aleatoriedad y la apertura a los sonidos del entorno son características de la obra de Cage. El vacío y la repetición son elementos que reenvían a la idea de boredom que Goldberg identifica como un concepto importante en el arte de la década de 1970: desde los motivos repetitivos en la música de Steve Reich y Phillip Glass, hasta los gestos reiterados en la danza de Trishia Brown (Goldberg, 1995, p. 17). La idea del vacío también recuerda la impronta de Yves Klein, cuya influencia suele ser mencionada por Abramović (lles, 1996, p. 22).

Por último, quisiera hacer una breve mención al sonido en las videoperformances que siguieron a la serie Rhythm, entre las que se destacan Art Must be Beautiful, Artist Must be Beautiful (1975) y la serie Freeing (1975-76). Recordemos que, a diferencia del film, el dispositivo del videotape permitía la grabación conjunta de imagen y sonido, aun cuando la calidad fuera un tanto precaria. Es justamente la tosquedad de la toma de audio sin editar lo que permite situar a la audiencia en la inmediatez de la performance. Por ejemplo, en Art Must be Beautiful oímos el cepillo y la peineta agrediendo el cuero cabelludo de la artista junto al sonido de su voz. La fuerza de ambos sonidos, mezclada con aquellos del ambiente, como el ruido de coches, pone de manifiesto tanto el "aquí y ahora" de la acción, como su carácter auto lesivo de modo más evidente que la sola imagen de video. Si hacemos el ejercicio de ver la cinta sin sonido, no percibimos la violencia del gesto con la misma intensidad. Ésta queda de manifiesto a través de la escucha porque nos confronta con el sonido de la carne agredida, un sonido que parece resonar en nuestros propios cuerpos. Esto sugiere que aun cuando la acción se encuentre mediada por el video, el sonido parece restituir algo de las sensaciones físicas que interpelan a la audiencia en el momento de la performance.

\section{Del sonido a la música: el giro autobiográfico}

Tras poner fin a su relación artística y sentimental con el artista Ulay (Frank Uwe Laysiepen) en 1988, Abramović inicia una nueva etapa caracterizada por un giro hacia la autobiografía y hacia el teatro. En contraste con las instalaciones sonoras, performances y vídeos realizados en solitario durante la década de 1970, las piezas de la década de 1990 exploran elementos teatrales tales como la puesta en escena, el uso de vestuario y maquillaje, la realización de acciones prescritas en un guion, el empleo de proyecciones de video en paralelo a la acción, y el uso de música en lugar de sonido.

La exploración de estos elementos se aprecia en obras como Biography (1993) y Delusional (1994), ambas realizadas en colaboración con el videoartista americano 
Charles Atlas. Como parte del proceso de recopilación de información autobiográfica, Abramović regresó a Belgrado en 1994 para entrevistar a sus padres, generando un material de vídeo que fue empleado en Delusional y más tarde en Balkan Baroque (1997) (Westcott, 2010, p. 235). Ambas piezas se enmarcan en un proceso en el que la artista emplea la categoría de lo "balcánico" para referirse a la antigua Yugoslavia, algo que va acompañado por uso de músicas tradicionales del sudeste de Europa. En los siguientes apartados voy a centrarme en estas dos piezas.

\subsection{Delusional}

Delusional fue estrenada en Frankfurt en 1994 y se divide en cinco actos: "The Mother", "The Rat Queen", "The Father", "The Rat Disco" y "The Conclusion", cada uno aborda temas diferentes con una escenografía particular. De acuerdo a las descripciones de la pieza la música estaría presente en dos ocasiones (Stiles, 2016, p. 218; Richards, 2010, pp. 25-26). En la primera sección "The Mother", en el escenario había una cama de hielo, otra de metal, un taburete y una silla de metal, y el suelo estaba cubierto de ratas de plástico que sonaban al aplastarlas. Abramović entraba al escenario bailando frenéticamente al ritmo de piezas folclóricas húngaras, cuyos títulos no son aportados, y cada cierto tiempo se dejaba caer sobre la cama o la silla. Luego se proyectaban fragmentos de la entrevista a la madre de la artista, quien relataba algunos episodios de su vida mientras Abramović en el escenario narraba situaciones personales. Otro momento sonoramente relevante estaría en la cuarta sección "The Rat Disco", donde la plataforma transparente del escenario contenía ratas vivas que se convertían en las protagonistas de la sección. De acuerdo con las descripciones, una música bailable comenzaba a sonar con un volumen muy alto, haciendo que las ratas se movieran desorientadas. En este caso la música era empleada como una fuerza agresiva, un recurso que recuerda a la instalación Sound Corridor / War (1972).

Charles Atlas manifestó una visión crítica sobre esta colaboración, señalando que Abramović prefería regodearse en la vergüenza ante el conflicto bélico en los Balcanes sin ir más allá. De hecho, Atlas habría propuesto el título Delusional a modo de crítica a la actitud simplista que Abramović tenía de Yugoslavia y los Balcanes, algo que, según Atlas, la artista no habría entendido: "'Delusional was my title' [...] 'She liked the way it sounded. I don't think she knew what it meant, really." (Atlas entrevistado en Westcott, 2010, pp. 237-238)?].

Esta pieza suele ser considerada uno de los antecedentes de Balkan Baroque debido a que contiene algunos de sus temas y materiales de base: las canciones folclóricas, la imagen de los padres y las ratas. Pero, sobre todo, la pieza prefigura la imagen arquetípica los Balcanes que Abramović desarrollará más tarde: una tierra donde las emociones contrapuestas bailan al ritmo de músicas festivas, mientras hacen las cuentas con un pasado familiar marcado por el cristianismo ortodoxo y el comunismo. 


\subsection{Balkan Baroque}

Balkan Baroque fue presentada en junio de 1997 en la 47 Bienal de Venecia y Abramović fue galardonada con el León de oro a la mejor artista. Suele ser considerada una de las piezas más políticas de la artista por sus referencias a las guerras de Yugoslavia. ${ }^{5}$ Como mostraré a continuación la obra funciona como una metáfora muy desdibujada del conflicto bélico y el empleo de la música juega un rol en este proceso.

La pieza consistía en una videoinstalación en tres canales dispuestos a manera de tríptico $^{6}$. A la izquierda se proyectaba el busto del padre, a la derecha el de la madre imágenes que ya habían aparecido en Delusional-, y al centro la imagen de Abramović relatando cómo se crea la rata-lobo en los Balcanes llevando gafas y una bata blanca. Acabado el relato, se quita la bata y queda ataviada con un vestido negro mientras baila al ritmo de una canción-danza.

En medio del tríptico de vídeos, se encontraba Abramović sentada sobre una pila de huesos de res con restos de carne adherida. Durante 4 días la artista vestida con una especie de túnica blanca limpiaba estos huesos con un cepillo de dientes metálicos que remojaba en un cubo de agua. Mientras limpiaba, cantaba algunas melodías de canciones populares o a veces lloraba. El sonido rítmico del cepillo se mezclaba con su voz y con los sonidos procedentes de la videoinstalación.

La acción de limpiar huesos putrefactos provocó un fuerte impacto emocional en la audiencia, y autoras como Stiles ven en este gesto una referencia a la limpieza racial perpetrada por Serbia (Stiles, 2016, p. 213). Sin embargo, la propia Abramović ha señalado que para ella lo importante era crear una imagen atemporal en contra de la guerra $^{7}$. La elección de los elementos musicales que conforman la pieza y la información que se omite sobre ellos, parece estar enfocada a construir esta imagen que evade cualquier referencia específica.

5 Tras la disolución de la antigua Yugoslavia se sucedieron varios conflictos en la región, entre los que se pueden destacar: la guerra de independencia croata (1991-95), la guerra de Bosnia Herzegovina (1992-95) y la guerra de Kosovo (1996-99), la que coincide con la realización de Balkan Baroque.

6 Véase parte de la documentación audiovisual de la pieza en:

http://www.li-ma.nl/site/catalogue/doc/marina-abramovic/balkan-baroque-registratie-biennaledi-venezia/6849 (Consultado el 10/03/21)

7 "[...] for me was very important to create something which is universal. Because if you make a political piece just for the political action of the time next day is old news, like old newspaper, But I want to create an image that you can use for any war, any time in the world. So now there is no more war in Yugoslavia, but you can use the image for Syria ... or whatever war is going to be in the future. (Abramović entrevistada en Lund, 2017)? 
Sobre las canciones populares vocalizadas por Abramović hay poca información. La documentación audiovisual disponible no permite identificar las melodías con claridad y en la descripción de la obra en los diferentes catálogos sólo se aporta la traducción de los primeros versos (Abramović et al, 1998, p. 364). La autora Bojana Pejić, quien se habría entrevistado con Abramović durante la Bienal de Venecia, señala que la artista pretendía cantar cada día una canción representativa de alguna de las zonas que conformaban la antigua Yugoslavia ${ }^{8}$. En cuanto a la canción-danza usada en la videoinstalación tampoco hay claridad. Abramović ha descrito esta pieza de maneras muy diferentes, ya sea como "Hungarian Czardas" (The Museum of Modern Art, 2010) o "fast Serbian folk tune" (Abramović 2016). Algunos escritos posteriores la definen como "a folk melody, originating from eastern Serbia and Romania" (Pejić, 2002, p. 337).

Las músicas presentes en la pieza podrían haber ayudado a retratar la diversidad cultural de la antigua Yugoslavia, pero la ausencia de información contextualizada al respecto acaba reduciendo su uso a una denominación genérica ("música de los Balcanes") que termina invisibilizando la dimensión política específica que podría haber tenido la obra. Si nos limitamos a la imagen de la performance, nos quedamos con la puesta en escena de una infructuosa expiación y con el mensaje universal en contra del genocidio. Pero si la hubiésemos escuchado, tal vez nos habríamos confrontado con controversias específicas sobre el conflicto. Al no entrar en esta dimensión política, Balkan Baroque se presenta como una obra autobiográfica sobre los conflictos de la artista con su familia y su contexto de origen.

\section{Conclusiones}

En este texto he intentado mostrar cómo Abramović emplea el sonido para explorar algunos de los elementos que aparecen en sus primeras performances de la serie Rhythm y que continuarán madurando en el resto de su obra, tales como la temporalidad, la repetición y la saturación de los sentidos para luego vaciar la mente. También se destaca la escucha de sí y del entorno como una condición que permite explorar los límites del cuerpo. Entre las piezas de la década de 1970 y las de 1990 se advierte un cambio significativo. En la primera etapa la artista presenta el cuerpo de manera directa y el sonido era abordado como un material que parecía complementar esta aproximación radical a los límites del cuerpo. En la segunda etapa, en cambio, la

8 "The first day it was a Russian ballad from the Fifties, sung by a very popular Yugoslav woman singer; second, she sung a city ballad originating in Serbia; the third day it was a folk song from Dalmatia (Croatia). If the performance could last seven days, she stated, she would sing a melody from all other Yugoslav ex- republics, Bosnia and Herzegovina, Macedonia, Montenegro and Slovenia." (Pejić, 1998, 37) 
aproximación al cuerpo se orienta hacia lo teatral y el sonido pasa a un segundo plano. Abramović se centra en el cuerpo como soporte de la autobiografía y emplea músicas tradicionales del Sud Este de Europa para ilustrar su cultura de procedencia, algo que, si bien le permite vincular su subjetividad con la de una colectividad, acaba creando un cuadro pintoresco de los Balcanes, en el que diluye la problemática específica de las guerras en la antigua Yugoslavia.

Este estudio abre líneas de trabajo para indagar sobre la relación entre experimentación sonora, performance y video arte, tres prácticas que presentan como denominador común la temporalidad, y que han sido transitadas por diversos artistas desde la época de Abramović hasta la actualidad. Abordar su desarrollo conjunto puede arrojar información interesante para comprender algunas tendencias de la creación actual.

Para finalizar, creo que este estudio plantea la urgencia de prestar más atención a lo sonoro en el arte. Mientras galerías y museos se llenan de piezas con algún componente sonoro, parece ser que nuestra capacidad para escuchar las obras y construir discurso al respecto aún está en ciernes. Hace falta seguir reflexionando sobre cómo el sonido puede aportar nuevas ópticas de lectura para la investigación sobre el arte actual.

\section{Referencias}

ABRAmović, M. (2016). Walk through walls: a memoir. New York: Crown Archetype.

Abramović, V., Avgikos, J., lles, C., McEvilley, T., Obrich, H. U., Pejic, B., Wulffen, T. (1998). Marina Abramovic: The Artist Body. Performances 1969-1998. Milano: Charta.

BiesenBACH, K. (2010). Marina Abramovic. The Artist is present. The artist was present. The artist will be present. En Marina Abramovic: The Artist is Present (pp. 12-21). New York: Museum of Modern Art.

BIESENBACH, K. (2016). The Fundamentals of Endurance: Marina Abramović on How She Learned to Refuse the Body's Limits and Make Immortal Art. Recuperado 13 de diciembre de 2018, de https://www.artspace.com/magazine/interviews_features/book_report/marinaabramovic-interview-klaus-biesenbach-54182

BiesenBaCH, K., Stokić, J., Danto, A. C., \& Iles, C. (2010). Marina Abramović: The Artist is Present. New York: Museum of Modern Art.

Brayshaw, T., \& Witts, N. (2013). The Twentieth Century Performance Reader. Oxon \& New York: Taylor and Francis. 
Cuerpo, límites y autobiografía: Una aproximación a lo sonoro en la obra de Marina Abramović

FISCHER-LICHTE, E. (2011). Estética de lo performativo. Madrid: Abada Editores.

Goldberg, R. (1995). Here and Now. En Marina Abramović: Objects, Performance, Video, Sound (pp. 11-18). Oxford: Museum of Modern Art Oxford.

HegartY, P. (2015). Rumour and Radiation: Sound in Video Art. London \& New York: Bloomsbury Publishing.

ILES, C. (1995). Cleaning The Mirror. En Marina Abramović: Objects, Performance, Video, Sound (pp. 21-34). Oxford: Museum of Modern Art Oxford.

ILES, C. (1996). Marina Abramovic (interview). Performance Research, 1(2), 20-26.

LABELLE, B. (2006). Background noise: perspectives on sound art. Continuum International.

LABELLE, B. (2014). Lexicon of the mouth: poetics and politics of voice and the oral imaginary. New York \& London: Bloomsbury.

Lund, C. (2017). Marina Abramović Electricity Passing Through (interview). Recuperado 29 de julio de 2019, de https://channel.louisiana.dk/video/marinaabramovic-electricity-passingthrough

O'Hagan, S. (2010). Interview: Marina Abramović. Recuperado 8 de agosto de 2019, de https://www.theguardian.com/artanddesign/2010/oct/03/interview-marina-abramovicperformance-artist

Pejıć, B. (1998). Bodyscenes: An Affair of the Flesh. En Marina Abramovic: The Artist Body. Performances 1969-1998 (pp. 26-40).

PEJı́, B. (2002). Balkan for Beginners. En L. Hoptman \& T. Pospiszyl (Eds.), Primary Documents: A Sourcebook for Eastern and Central European Art Since 1950. (pp. 325-339). New York: The Museum of Modern Art.

PEŠLć, N. (2018). New Age Healing in Marina Abramović's Art. Esotericism, Literature, and Culture in Central and Eastern Europe, 241-257.

RICHARDS, M. (2010). Marina Abramović. New York: Rouletdge.

RICHARDS, M. (2018). Marina Abramović. Routledge.

SKC, S. kulturni centar B. (2009). Ovde, na ovom mestu Marija Abramović je... Recuperado 15 de agosto de 2019, de https://www.skc.org.rs/abeceda-skc-a/ovde-na-ovom-mestu-marijaabramovic-je.html 
Úrsula San Cristóbal

StILES, K. (2016). Cloud with its shadow: Marina Abramovic. En Concerning Consequences: Studies in Art, Destruction, and Trauma. London \& Chicago: University of Chicago Press.

The Museum of Modern Art. (2010). Marina Abramović: The body as medium. Recuperado 8 de enero de 2021, de https://www.youtube.com/watch?v=OUrwdqwzqMU

WestCotT, J. (2010). When Marina Abramović dies: a biography. Cambridge: MIT Press. 